Dawid Winiarski*

\title{
The use of EMG signal in human-machine interface
}

\section{Introduction}

The dynamic development of electronics, mechanical engineering, and biomedical engineering has opened up new possibilities in the field of prosthetics and smart devices. Thanks to modern equipment, rehabilitation is faster, more-advanced surgery is possible, and recovery after accidents is more efficient. The observation of such bioelectric signals as EEGs, ECGs, and EMGs is often the basis for finding a particular disease or deciding on further treatment(s) for the patient. Research related to biomedical engineering and processing bioelectric signals is still ongoing. There are more and more devices controlled by human-machine interfaces. To improve such systems, more-accurate measurement systems, more-complex control algorithms, and more-precise positioning systems are needed. The topic of discussion in the following article will be the analysis of EMG signals, which is the process of forming electrical potentials on human skin during muscle tension (specifically, surface EMG [sEMG]). This signal is mainly used for testing motor dysfunction among people. The aim of the study is to present the problem of measuring and filtering an EMG signal. Through the use of digital filtering and the appropriate signal processing, relevant information carried by the signal should be obtained. The experiment will provide the results on whether an EMG signal is suitable for use in systems controlled by muscle tension (for example, an intelligent prosthetic hand or exoskeleton). The following article has been mainly based on position [7]. Many content and drawings were taken from it.

\section{Biological aspects of the EMG signal}

This section is mostly based on position [3].

"Electromyography (EMG) is a valuable technique for studying human movement, evaluating mechanisms involving neuromuscular physiology, and diagnosing neuromuscular disorders. However, there are many potential pitfalls in the use of EMG as a tool.

* AGH University of Science and Technology, Department of Automatics and Biomedical Engineering, Krakow, Poland 
The question that a researcher is asking may not be amenable to solution using EMG techniques. (...) Furthermore, the interpretation of the EMG signal requires a thorough knowledge of the origin of the signal." The waveform of an EMG signal is frequently evaluated as an electrical signal; this is why its characteristics can be analyzed using conventional signal processing techniques. The source of an EMG signal is a single muscle fiber or group of fibers. The anatomical features of an individual fiber and physiology of the whole muscle action potential formation are key to understanding how to record, analyze, and interpret the EMG signal.

While at rest, there is a potential gradient across the membrane of the muscle fiber. Inside the cell, there is the potential of about $-90 \mathrm{mV}$ with respect to the exterior of the cell. The potential difference is produced with different concentrations of sodium cations $\left(\mathrm{Na}^{+}\right)$, potassium cations $\left(\mathrm{K}^{+}\right)$, chlorine anions $\left(\mathrm{Cl}^{-}\right)$, and other anions near the membrane. During the state of rest, the concentration of $\mathrm{Na}^{+}$ions is relatively high on the outside of the cell membrane and relatively lower inside. On the other hand, the concentration of $\mathrm{K}^{+}$ ions is relatively low on the outside and higher inside the muscle fiber. Muscle fibers are excitable tissues. When the fiber is depolarized by a potential of about $10 \mathrm{mV}$ or greater, the membrane potential reacts specifically and in a predictable manner to produce a response that is called the muscle fiber action potential or simply action potential.

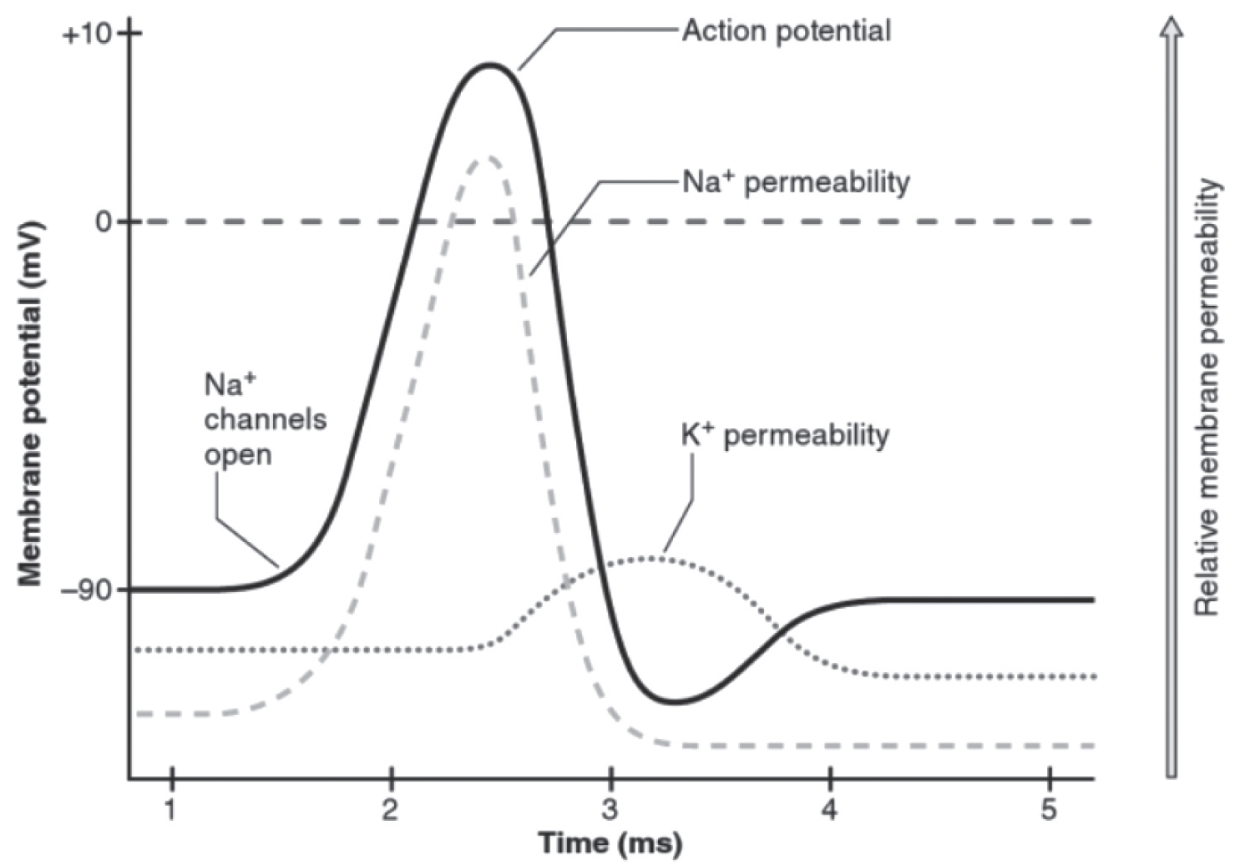

Fig. 1. The time course of muscle fiber action potential is mediated be changes in membrane permeability to $\mathrm{Na}^{+}$and $\mathrm{K}^{+}$ions [3] 
The action potential is generated through a neuromuscular joint and spreads along the muscle fiber in both directions relative to this joint. In the first phase of the action potential, the permeability of sodium cations grows and move into the cell, eventually reversing the polarity of the cell so that it temporarily reaches a positive potential - about $+10 \mathrm{mV}$. When the migration of $\mathrm{Na}^{+}$increases, then the membrane permeability for $\mathrm{K}^{+}$ is changed. They emerge on the outside, which eventually results in the potential return to the resting state. Described process of generation of action potential has been presented in Figure 1.

A motor unit is described as a combination of a single motoneuron and all of the muscle fibers innervating by this motoneuron. All fibers that the motoneuron innervates are activated at the same time as when action potential appears on the motoneuron. Bearing in mind that many individual muscle fibers are innervated by the same motoneuron in any motor unit, each of these fibers generate a discharge almost simultaneously.

The total activity of the muscle fibers of the same motor unit results in generation of motor unit action potential (MUAP). The signal amplitude of a motor unit is the superposition of all action potentials generated by the muscle fibers. This process is shown in Figure 2.
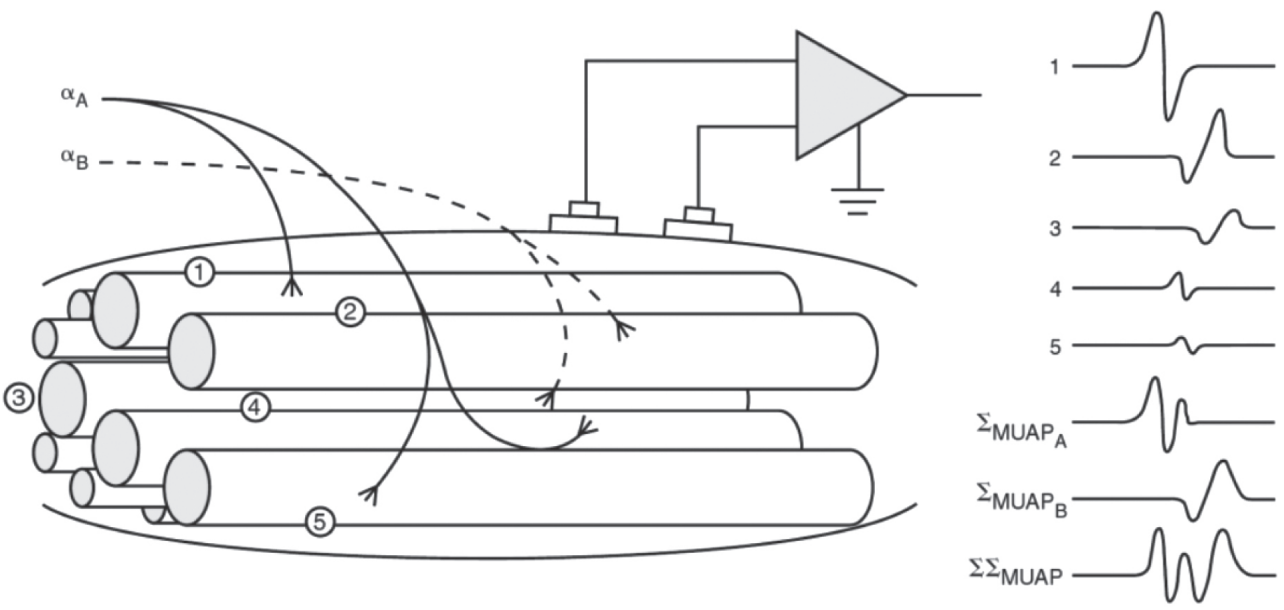

Fig. 2. The surface electromyogram as a composition of signals form all motor unit action potentials [3]

\section{Amplifier of the biopotentials}

As the amplifier of biopotentials, an instrumental amplifier has been applied. This is a combination of non-inverting amplifiers and a differential amplifier. It combines the positive attributes of both applications. A schematic diagram is shown in Figure 3. 


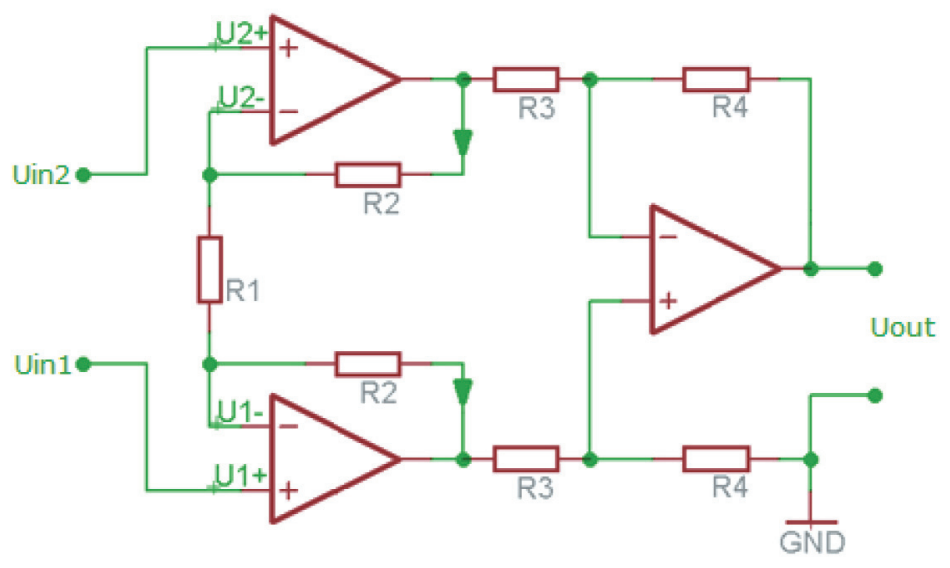

Fig. 3. Schematic diagram of the instrumental amplifier

The signal is given separately on the two non-inverting input operational amplifiers, which guarantees the very high input impedance of the circuit. In addition, the output of the operational amplifiers are connected to the differential amplifier. Therefore, measurement of the inputs is differential. The overall gain of the system can be represented by formula (1):

$$
k=\frac{R_{4}}{R_{3}}\left(1+2 \frac{R_{2}}{R_{1}}\right)
$$

The expression above was explained in [9]. This system is more resistant not only to additive interferences but also to changes in temperature.

The application of the EMG amplifier was based on the INA128 chip of Burr-Brown [1]. INA128 is an instrumental amplifier as previously described. The gain of the system is determined by the selection of an appropriate resistor value. In this case, the gain was set to $k=1000$.

Basic features of the biopotentials amplifier [6]:

- The high value of the gain due to the very subtle nature of bioelectric signals.

- The frequency band of the amplifier should be correctly chosen to include all of the frequencies of the measured signal. This is determined by the lower and upper frequency limits.

- The Common Mode Rejection Ratio, defined as the ratio of differential signal amplification to summation signal amplification.

- Noise and drift phenomenon cause incorrect readings of measured values.

- High input impedance of the amplifier. 


\section{Digital filtering of the signal}

Processing the raw EMG signal is practically impossible; therefore, the proper filtering of the signal is very important. The use of correctly designed filters can remove or significantly reduce the volume of unwanted and unexpected noise. The spectral power density of a surface EMG signal has an insignificant band of spectrum beyond the scope of $5-10 \mathrm{~Hz}$ to $400-450 \mathrm{~Hz}$. The bandwidth of the amplifier/filter should be within this range; for example, $5 \mathrm{~Hz}$ for the high-pass filter and $500 \mathrm{~Hz}$ for the low-pass filter [5]. Due to the given frequency limit values, a band-pass Butterworth filter has been designed in MATLAB. In addition, an interfering signal at a frequency of $100 \mathrm{~Hz}$ appeared during the experiments, probably as a harmonic of power line voltage. Accordingly, a band-stop filter has been implemented whose task is to eliminate this interference.

\section{Measurements}

An RT-DAC4 PCI INTECO multifunction analog and digital timing I/O board was used for data acquisition. The voltage resolution of the cart is $4.88 \mathrm{mV}$, and the sampling frequency was set to $5 \mathrm{kHz}$ [2]. The measurement of action potentials was conducted with dermal electrodes stuck to the surface of the skin, and shielded cables derived from the amplifier of biopotentials were attached to them. The investigated muscles and locations of the measuring electrode pairs have been marked in Figure 4.

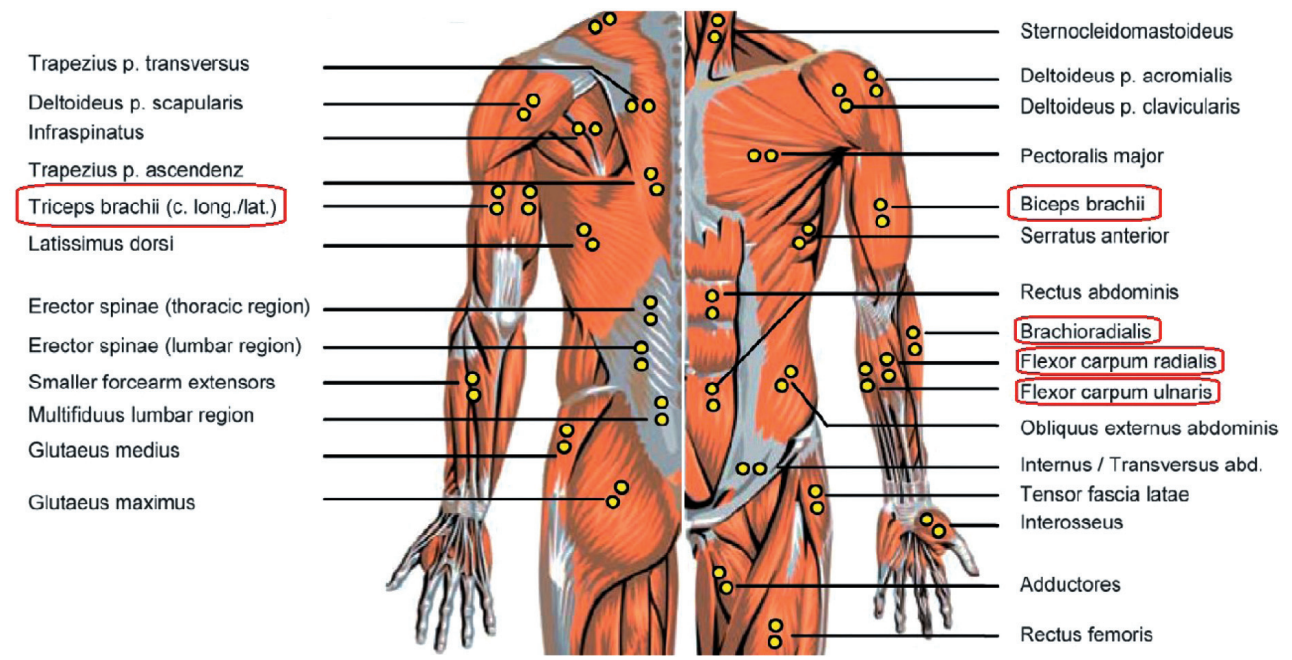

Fig. 4. The muscles in the human body. The back view (left) and front view (right). Based on [4] 
Each of the four channels of the amplifier corresponds to the studied muscle:

- Channel 1 - Biceps brachii muscle,

- Channel 2 - Triceps brachii muscle,

- Channel 3 - Flexor carpi ulnaris muscle, flexor carpi radialis muscle,

- Channel 4 - Brachioradialis muscle.

During registration, the test person has performed a variety of arm movements. Figure 5 shows electrode location applied on the arm and a prototype of the four-channel amplifier of biopotentials. The raw EMG signal is presented in Figure 6. In this case, the motion was bending the arm at the elbow.

It can be seen that the signal is very noisy and cannot be processed further in this form. During careful analysis, it turned out that in the measurement there was an interfering sine signal at a frequency of $100 \mathrm{~Hz}$ and amplitude reaching up to $1.5 \mathrm{mV}$ (which is the harmonic of the power supply). As previously mentioned, the distortion was filtered by a band-stop filter. It may be noted that low frequency noise appeared in Channel 1 of the amplifier. In this type of interference, a band-pass filter with a lower cut-off frequency of $5 \mathrm{~Hz}$ should reduce this effect. The results after applying the designed filters can be seen in Figure 7. The filtration process enables the extraction of all relevant features in the EMG signal on all four channels of the amplifier. Oscillations at the beginning of the plots are the effect of using a relatively high-order filter and should be rejected in further analysis [7].

a)

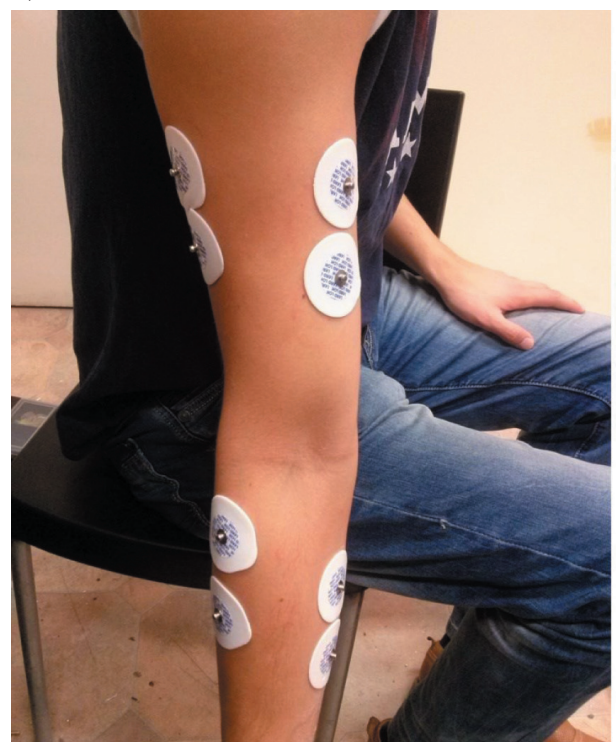

b)

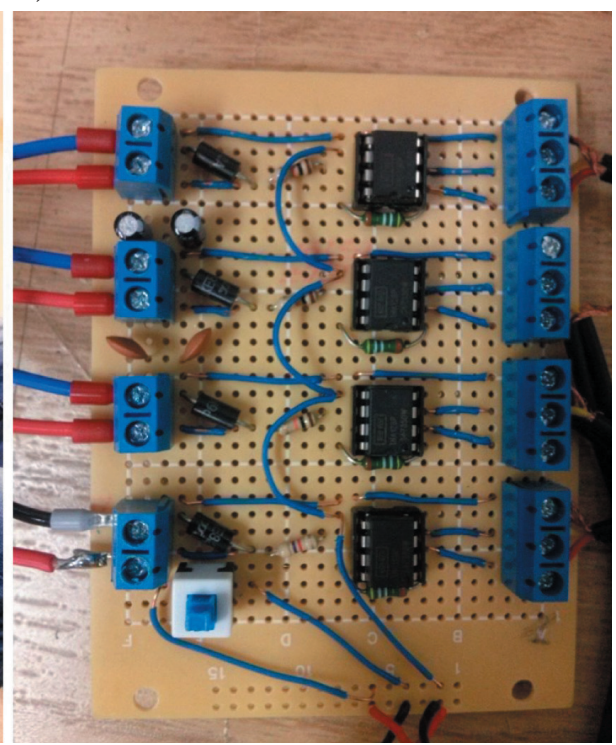

Fig. 5. Electrode location on the arm (a) and prototype of four-channel amplifier of biopotentials (b) 

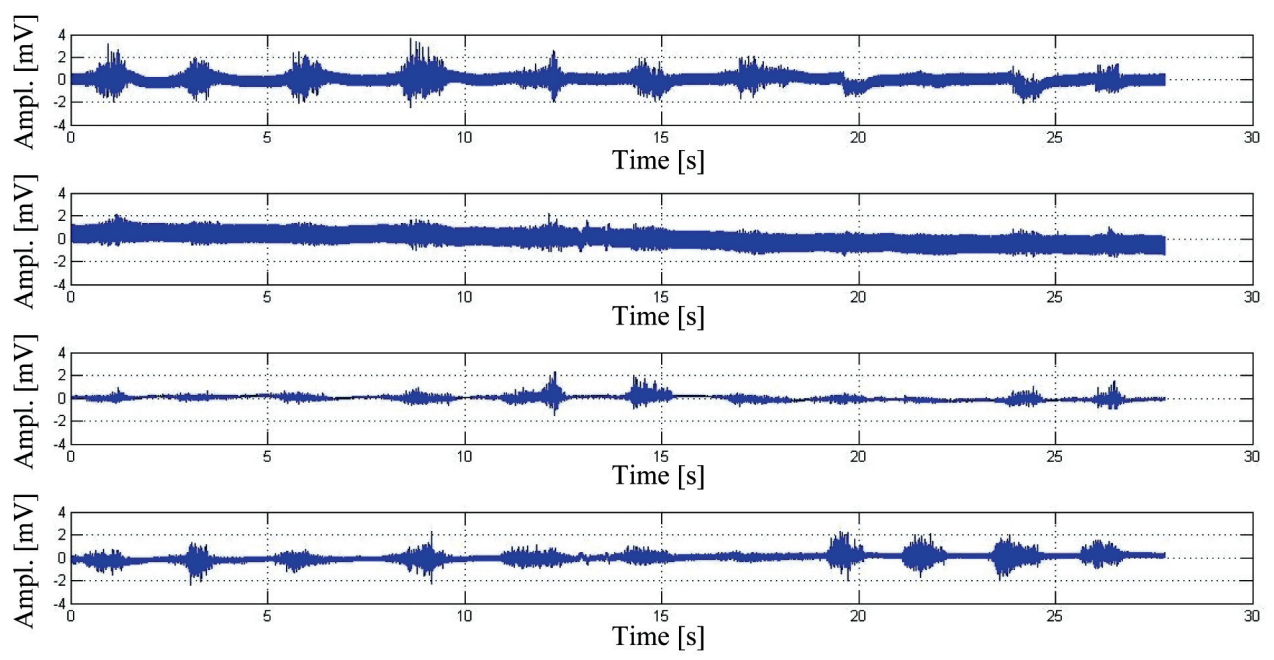

Fig. 6. Raw EMG signals recorded on four channels (first channel is up)
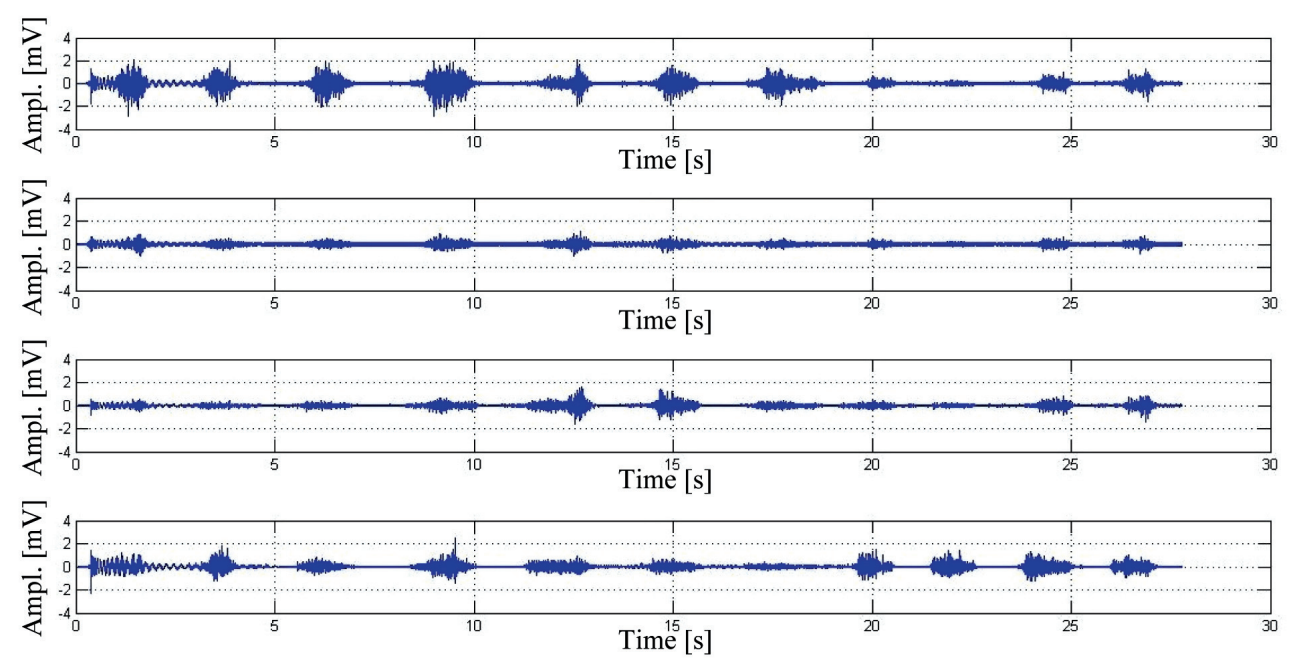

Fig. 7. Filtered EMG signals

While analyzing the EMG signal, several important things should be observed. First of all, the signal amplitude for the first channel is higher (where bioelectric activity of the biceps was recorded). This is connected with the fact that the biceps muscle is relatively big. In addition, this muscle works the most in the case of such a specific movement as bending the arm at the elbow. Another feature of the EMG signal is the increasing signal amplitude with higher muscle activity. In the state of rest, the signal value is close to zero. When the activity of a muscle increases, the amplitude of its EMG signal grows. 


\section{Analysis in the time domain}

The recommended method of analysis in the time domain of the EMG signal amplitude is to monitor changes over time [4]. The signal processing is to reverse the negative signal values to positive - this is the equivalent to calculating the absolute value of the signal. In addition, the next step is averaging the signal through low-pass filtering. The most-common algorithms are Moving Average and Root Mean Square.

The output values of the first algorithm (Moving Average) is calculated by the following formula (2):

$$
y_{M A}(k)=\frac{1}{2 N+1} \sum_{i=k-N}^{k+N} x(i)
$$

where:

$y_{M A}(k)-k$-sample of filtered signal (Moving Average),

$x(i)-i$-sample of original signal,

$2 N+1$ - width of the filtration window (number of samples).

The second algorithm (Root Mean Square) calculates the output similarly, but with slight differences:

$$
y_{R M S}(k)=\sqrt{\frac{1}{2 N+1} \sum_{i=k-N}^{k+N} x(i)^{2}}
$$

where:

$$
\begin{aligned}
y_{R M S}(k) & -k \text {-sample of filtered signal (Root Mean Square), } \\
x(i) & -i \text {-sample of original signal, } \\
2 N+1 & - \text { width of the filtration window (number of samples). }
\end{aligned}
$$

Now analyzed was the only part of the EMG signal from the biceps of channel 1. In the first step, the absolute value of the signal should be computed. In the next step, the signal should be normalized. Normalization is accomplished by dividing the total signal by the maximum value. The effect of this operation is shown in Figure 8.

The final stage of EMG signal processing is smoothing it with a low-pass filter. The recommended time-for-filtration window is a window with a width from $10 \mathrm{~ms}$ to $250 \mathrm{~ms}$ [5]. The sampling period is $5 \mathrm{kHz}$, so the window with a width of $10 \mathrm{~ms}$ is accomplished through the use of 50 consecutive samples, and a 250 -ms window corresponds to 1250 samples. It was decided that a time window of $30 \mathrm{~ms}$ will be used. This means calculating new values of the signal on the basis of 150 samples (the number of samples in a centered average should be an odd value; therefore, 151 samples were used in this window). The effect of the filtering is shown in Figure 9. 

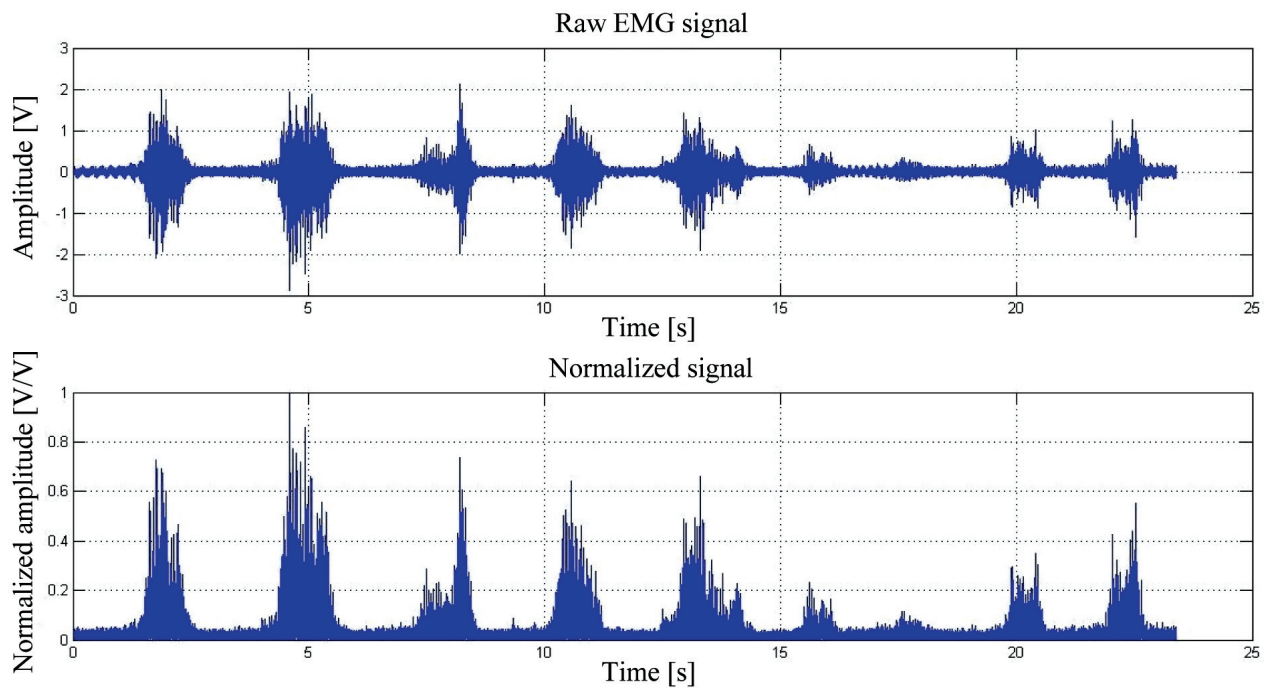

Fig. 8. Raw EMG signal (above) and normalized signal (below)
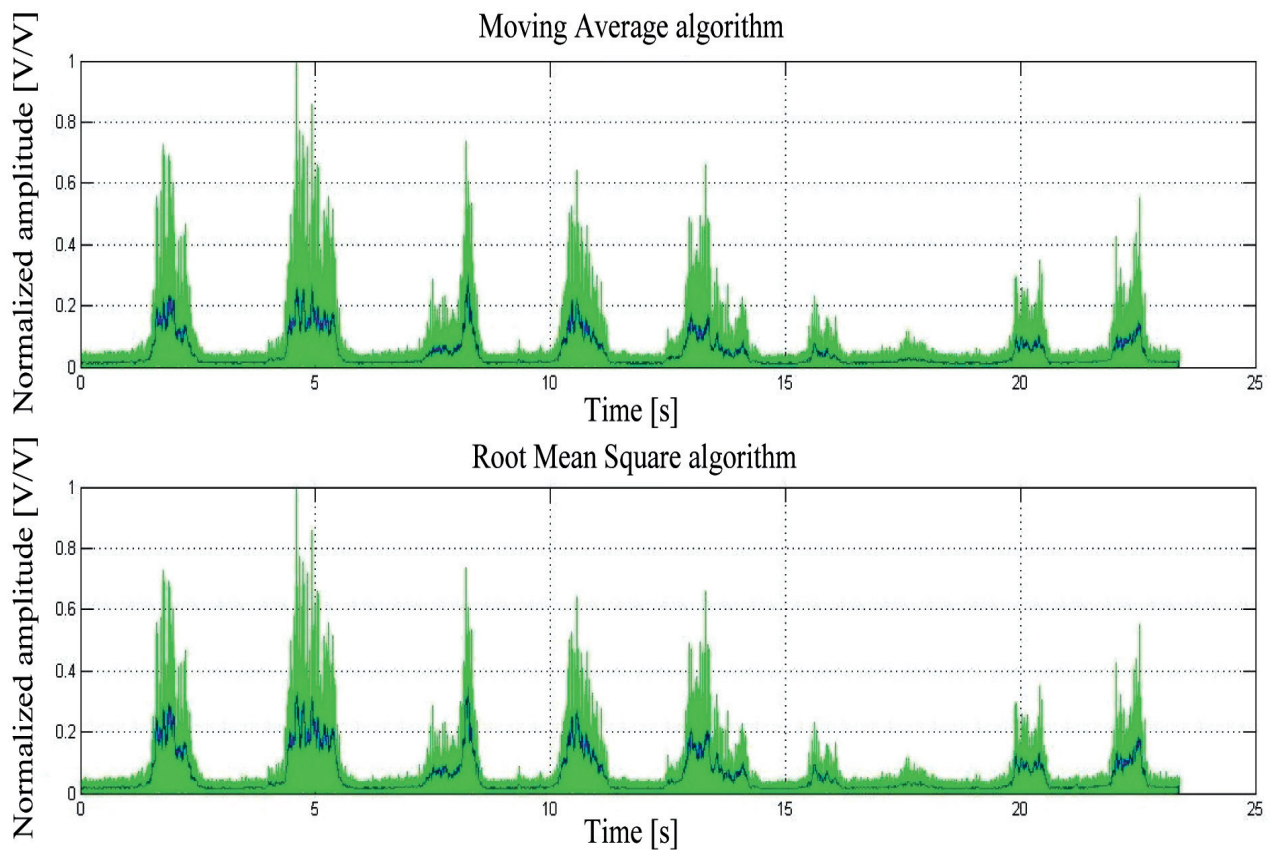

Fig. 9. Smoothing normalized signals (green plots) with moving average algorithm (above) and root mean square algorithm (below) 
Both graphs look very similar, but it can be seen that the amplitude of the received signal is slightly higher for the RMS algorithm. For smoothing the records, a RMS smoothing filter is recommended, as the results reflect the power of the signal. It should be noted that the Moving Average algorithm requires less computational effort than the Root Mean Square. These are very important issues when building a real-time processing system [7]. Such a processed EMG signal may be the basis for a control system for an artificial arm or support for the work of an exoskeleton. The increase in amplitude in the EMG signal indicates the desire to do the specific movement (which is, for example, bending the arm). In the simplest system, it can be used an ordinary comparator that will monitor the RMS value of the EMG signal from muscles. If the amplitude of the signal exceeds the threshold value, the system reacts the modulation motor or another actuator. The functionality of such a system can be seen in Figure 10 .
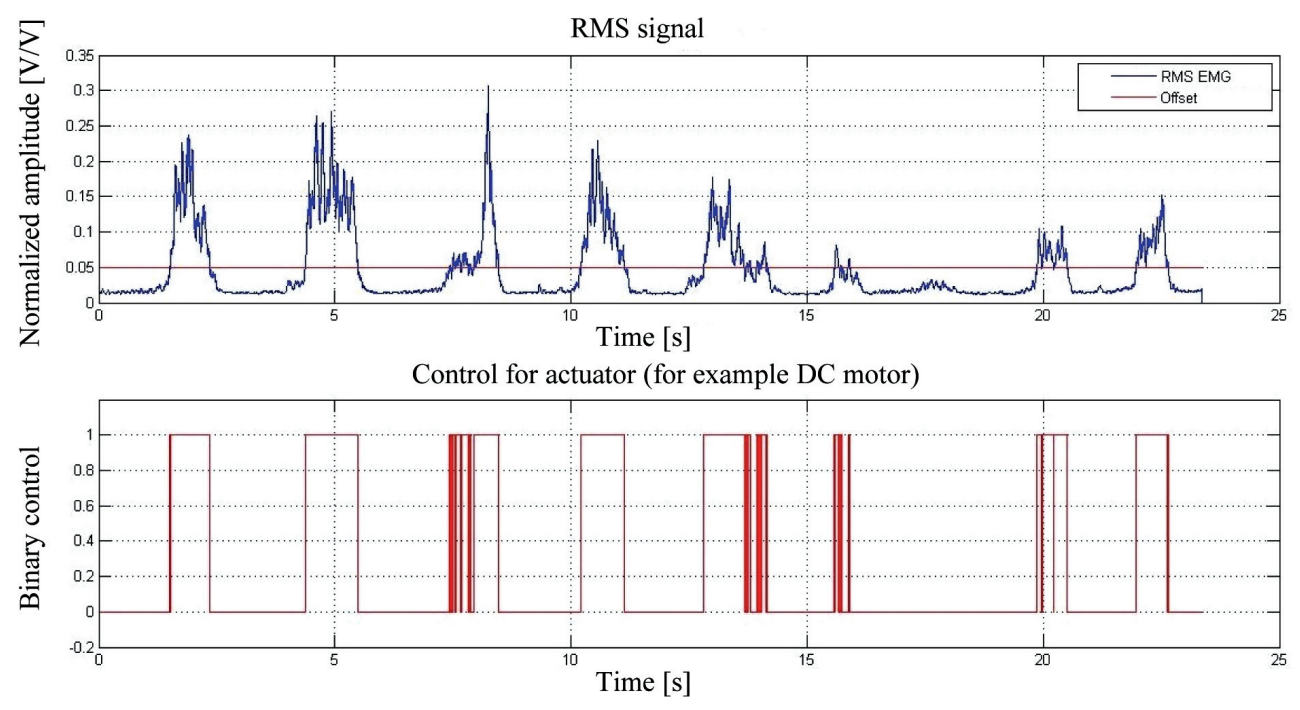

Fig. 10. Motor control with amplitude of EMG signal (RMS filter)

\section{Analysis in frequency domain}

The analysis of the EMG signal in the frequency domain will be based mainly on the change of the signal's spectrum over time. Calculating the Fourier transform of the whole 25 -second waveform is pointless. At what time the window should be set for which frequency spectrum will be determined. The fixed width of the window is 1000 samples, which corresponds to a period equal to $200 \mathrm{~ms}$. In Figure 11, there a summary of the EMG signal spectral changes is presented in the activity and passive states. 

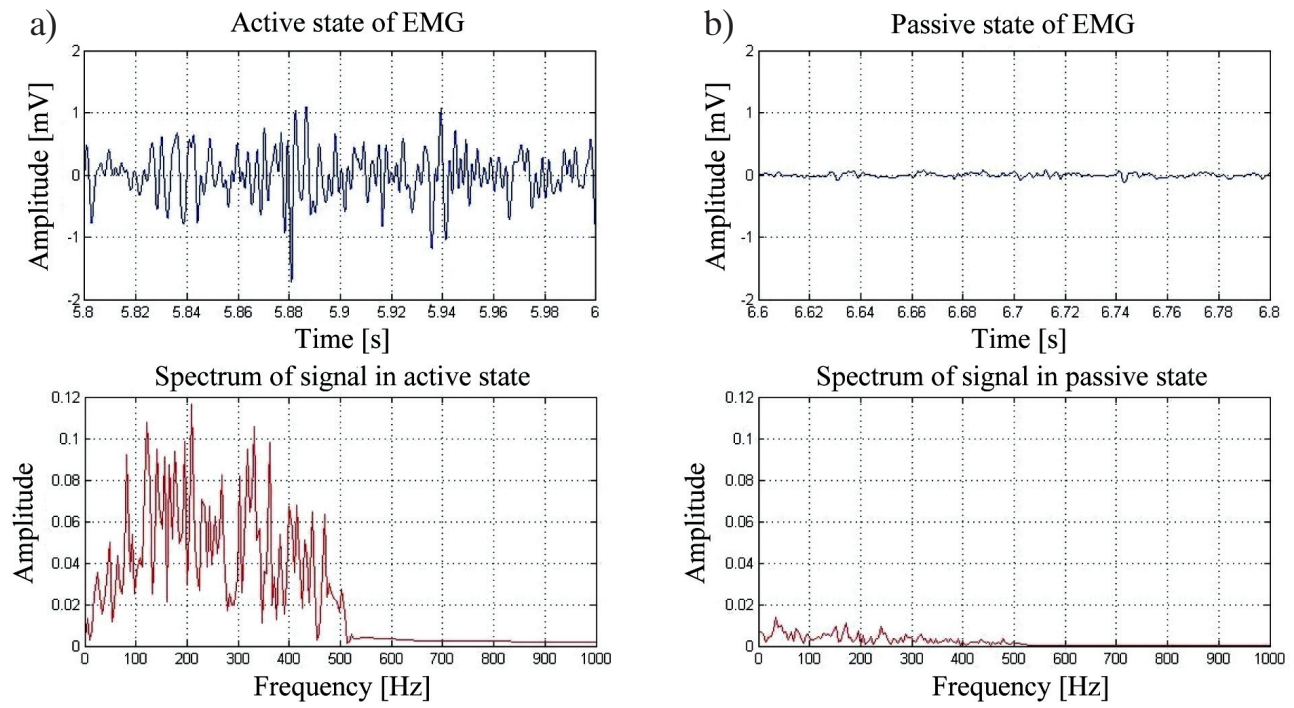

Fig. 11. View of spectrum of the signals in passive state (a) and passive state (b)

A relative increase in the signal amplitude in the time domain results in an increase of the value of individual bands of the spectrum in the frequency domain. The shape of the spectrum of the signal can provide important information about the activity of the muscle. In the passive state, the spectrum provides information about the EMG signal during rest. It is not possible to completely overcome the muscle activity. Another issue is the fact that some muscles work without human awareness (for example, the muscles of the heart). A passive EMG also gives information about the level of noise. In the control of artificial prosthesis, a passive spectrum can be used as a base. It should be the averaged spectrum of all recorded images of the passive state to determine the spectral pattern. This pattern could indicate whether the current spectrum points to activity or not.

After determining the spectrum for a given window, some parameters should be specified. Four parameters will be presented: integral of spectral density function, average frequency, median frequency, and maximum frequency.

$\left(I_{S D F}\right)$ - measure of total power transmitted by the signal. In the active state, signal power is relatively high; in the passive mode - low. This is calculated by the following formula (4):

$$
I_{S D F}=\int_{0}^{\frac{f_{S}}{2}} W(f) \cdot d f
$$

where:

$W$ - curve of the spectrum,

$f_{S}$ - sample frequency. 
Another parameter is the average of the curve of the spectrum $\left(f_{a}\right)$. This decides which frequency is statistically average. It is determined by the following formula:

$$
f_{a}=\frac{1}{I_{S D F}} \int_{0}^{\frac{f_{S}}{2}} f \cdot W(f) \cdot d f
$$

The next parameter is the median frequency $\left(f_{m}\right)$. This is defined as the frequency value for which the area under the curve of spectrum from 0 to this value is equal to half of the entire area:

$$
0.5 \cdot I_{S D F}=\int_{0}^{f_{m}} W(f) \cdot d f
$$

The last assumed parameter is the maximum frequency $\left(f_{\max }\right)$. This defines the frequency for which the amplitude of the spectrum is the highest. The pattern is shown below:

$$
f_{\max }=\max _{f \in\left(0 ; \frac{f_{S}}{2}\right)} W(f)
$$

All four parameters were calculated for the recorded EMG signal. The time window (width $200 \mathrm{~ms}$ ) was moved every $2 \mathrm{~ms}$ (10 samples) to the front to get a good resolution of the generated signals with relatively low CPU usage. The results of instantaneous spectral EMG parameters are shown in Figure 12.

In the second graph, it can be seen that the total power of the signal increases with the amplitude of the EMG signal. However, there is an apparent delay between the maximum amplitude of the EMG signal and maximum instantaneous power. The graph of the integral of the spectral density function is smooth and could be used as a processed EMG signal amplitude (from previous section) to control a motor in a system of intelligent prosthesis. Graphs of instantaneous total power of the EMG signal and control are shown in Figure 12.

Unfortunately, this approach also does not solve the problems related to the speed of the motor and its proper positioning. Moreover, the computational effort required to determine the instantaneous values of the area under the graph of the spectrum increases. This can introduce a significant delay in the system.

The graphs of average frequency and median frequency in Figure 12 look very similar. It may be noted that the instantaneous value of the median frequency changes a little more rapidly than the instantaneous average and achieves more-extremal values. During 
the active state, both increase to levels of about $250 \mathrm{~Hz}$, which indicates that a greater contribution in the EMG signal has higher frequencies. During rest (passive state), the average decreases to about $200 \mathrm{~Hz}$ and a median value of about $180 \mathrm{~Hz}$. These signals can provide additional information to the control system, indicating that the components have a higher share in the total signal power.

The graph of maximum frequency is very unstable. The instantaneous values are changing very dynamically. Despite this fact, changes in the signal are quite similar to the course average and median. The instability of the plot results in the low utility of the maximum signal in the control bionic prosthesis or exoskeleton (Fig. 13).
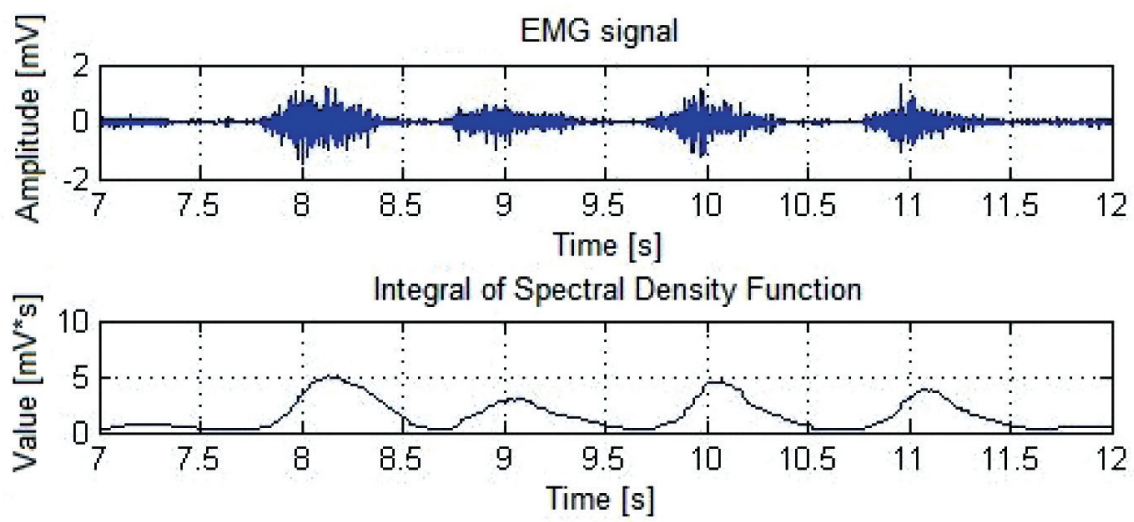

Average frequency

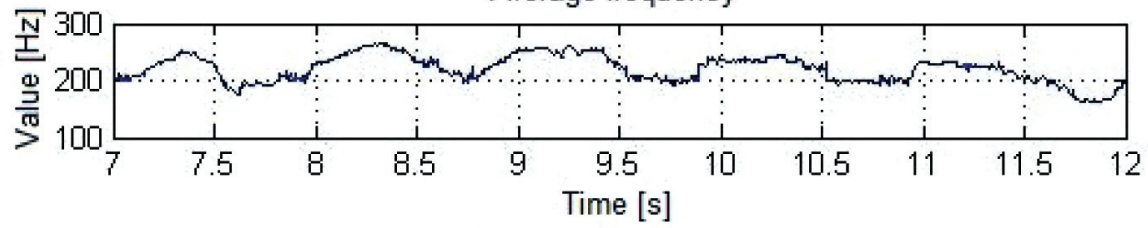

Median frequency

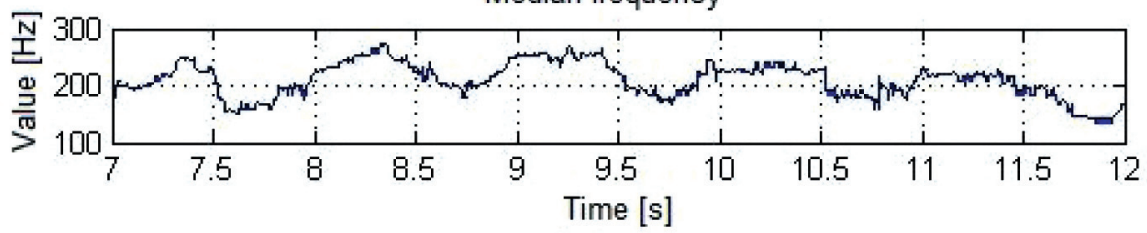

Maximum

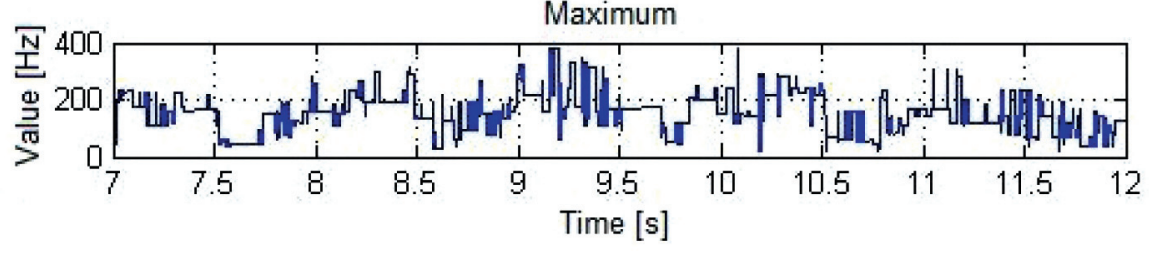

Fig. 12. Parameter of the spectrum of EMG signal 


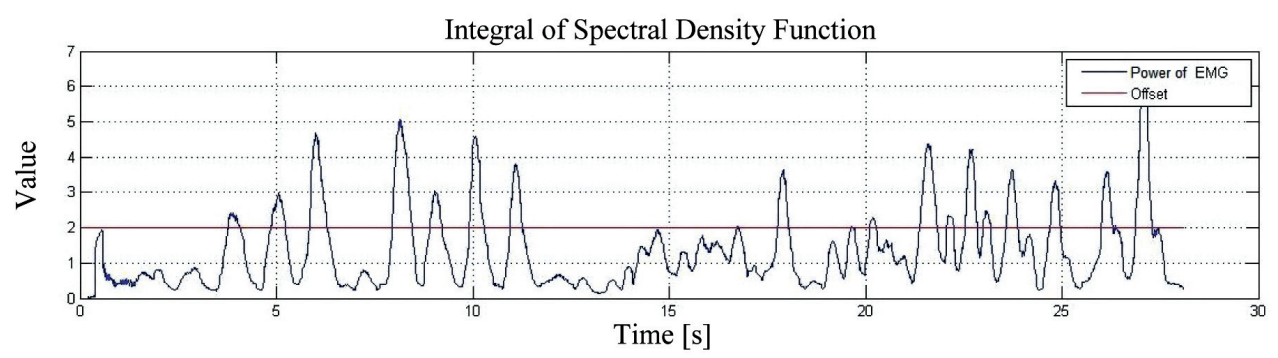

Control for actuator (for example, DC motor)

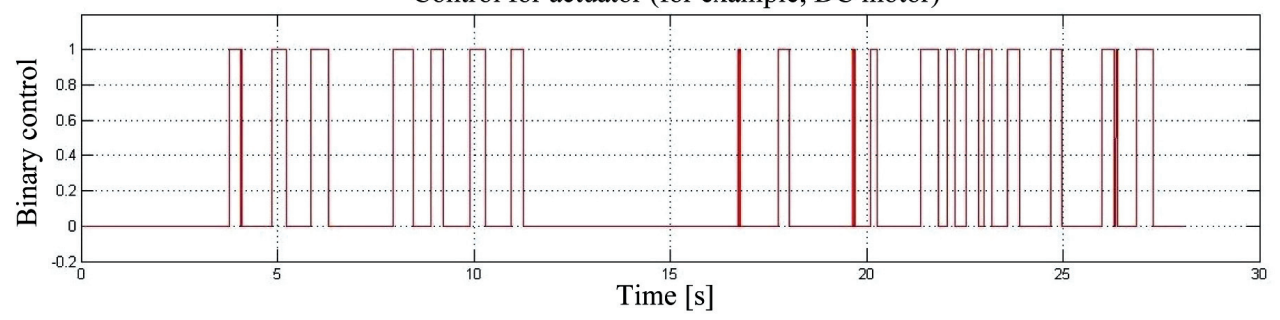

Fig. 13. Motor control with instantaneous power of EMG signal

\section{Summary}

In this article, the basic physiological phenomena that affect the formation of surface EMG signals has been described. Knowledge of these processes is necessary to properly and consciously analyze the registered signal. The high gain of the biopotential amplifier means that even the smallest external interference is transferred to the sampled signal. The use of a differential amplifier measurement in this application was appropriate because of the differential measurement, high input impedance, and low offset of the amplifier.

The implemented digital filters and scripts make it possible to remove interference measurements, process the signal, and extract the relevant signal parameters. Implementation of the EMG processing system would certainly differ from the offline analysis performed on the computer. Many aspects should be taken into consideration; the application certainly would impose time limitations, for example. This would be programmed on specialized equipment such as a microcontroller, whose task would be to continuously analyze the samples during the recorded signal and simultaneous generation of control for the bionic prosthesis or exoskeleton.

Controlling the manipulator with an EMG signal is not a simple task. It needs to be considered which signals from the muscles should be taken into account. The four measuring points proposed in this work seem to be reasonable and reflect the important muscles involved in the movement of the arm and the entire hand while minimizing the number of measurement electrodes. If the EMG signal is a superposition of the action potentials of all 
active motor units, it is desirable to know which unit affects which measured EMG signal. Consequently, if the measured EMG signal could be decomposed into signals (motor unit action potentials) from the respective motor units, interpretation of the EMG signal would be extremely simple. The problem remains difficult to solve. Indirectly, this has been investigated by the EMG signal spectrum, but such an analysis does not provide all of the necessary information.

In the case of control of a manipulator or exoskeleton system, problems arise with the positioning of the actuator. An EMG signal doesn't carry any direct information about the position of the shoulder. Another problem is connected with the direction of the motor. To resolve these issues, further studies and experiments should be carried out. Simple DC motor control using EMG signal amplitude seems to be achievable. It should be noted, however, that a manipulator designed in this way would not be able to accurately reproduce all of the movements of the human hand.

\section{References}

[1] Burr-Brown Products, INA128, INA129, Precision, Low Power INSTRUMENTATION AMPLIFIERS, USA 2000.

[2] INTECO, RT-DAC4/PCI Multi I/O Board Xilinx version User's Manual, Krakow 2002.

[3] Karmen G., Gabriel D.A., Essentials of Electromyography, USA 2010.

[4] Konrad P., ABC EMG, Praktyczne wprowadzenie do elektromiografii kinezjologicznej, Gliwice 2007.

[5] Merletti R., Standards for Reporting EMG Data, Torino 1999.

[6] Prutchi D., Norris M., Design and Development of Medical Electronic Instrumentation, New Jersey 2005.

[7] Winiarski D., Układ do pomiaru EMG, master's thesis, Krakow 2015. 